\title{
Enantioselective 5-exo-Fluorocyclization of Ene-Oximes
}

\author{
Taiki Rouno, Tomoki Niwa, Kousuke Nishibashi, Nobuharu Yamamoto, Hiromichi Egami $(\mathbb{D}$ and
} Yoshitaka Hamashima *iC

School of Pharmaceutical Sciences, University of Shizuoka, 52-1 Yada, Suruga-ku, Shizuoka 422-8526, Japan; s18125@u-shizuoka-ken.ac.jp (T.R.); s18405@u-shizuoka-ken.ac.jp (T.N.); m14085@u-shizuoka-ken.ac.jp (K.N.); m16117@u-shizuoka-ken.ac.jp (N.Y.); hegami@u-shizuoka-ken.ac.jp (H.E.)

* Correspondence: hamashima@u-shizuoka-ken.ac.jp; Tel.: +81-054-264-5672

Academic Editor: Norio Shibata

Received: 26 August 2019; Accepted: 23 September 2019; Published: 24 September 2019

\begin{abstract}
The enantioselective 5-exo-fluorocyclization of ene-oxime compounds was demonstrated under phase-transfer catalysis. Although deprotonative fluorinations competed, the chemical yields and the ee values of the desired isoxazoline products were generally moderate to good. The absolute stereochemistry of the major isomer was determined to be $S$ by comparison with the literature after transformation of the product to the corresponding iodinated isoxazoline.
\end{abstract}

Keywords: fluorine; asymmetric catalysis; phase-transfer catalysis; oxime

\section{Introduction}

Isoxazoline framework has been recognized as a significant substructure of natural and unnatural compounds [1,2], which possess various biological activities including anticancer activity [3,4], FXa inhibitory activity [5], and antiparasitic activity [6-9]. Therefore, the construction of isoxazoline has been actively studied [10-13]. Among various methods for isoxazoline synthesis, a difunctionalization-type cyclization of ene-oxime is one of the major strategies. Cyclization reactions with concomitant introduction of a fluoroalkyl group [14-17], a hydroxyl group [18,19], a thiocyanate group [20], and an iodine group [21] have been reported so far.

Fluorine chemistry has contributed to pharmaceutical and agrochemical sciences, because an introduction of fluorine atom(s) at an appropriate position often improves the property of the parent compounds in terms of metabolic stability, lipophilicity, and so on [22-27]. Thus, a tremendous amount of fluorination reactions, including the asymmetric versions have been investigated [28-30]. Reflecting that alkene is a useful feedstock in organic chemistry and easy to prepare, asymmetric fluoro-functionalizations of alkenes have attracted increasing attention [31-37]. However, there is no report of the asymmetric fluorocyclization of ene-oximes, while its racemic version was recently reported in 2017 [38].

Our recent research interests include the asymmetric fluorofunctionalization of alkenes by phase-transfer catalysis. In 2015, we reported the first successful example of asymmetric fluorolactonization of ene-carboxylic acids using a hydroxymethyl carboxylate phase-transfer catalyst [39]. Based on this study, we recently developed a linked-binaphthyl dicarboxylic acid precatalyst 1 , which was proven to be highly effective for the asymmetric fluorocyclization and the deprotonative fluorination of allylic amides (Scheme 1a) [40,41]. In these reactions, hydrogen bonding between the catalyst and the substrate was considered to be crucial for high asymmetric induction. Considering $\mathrm{pKa}$ values of amide and oxime, we anticipated that oxime could interact with our anionic phase-transfer catalyst through hydrogen bonds, which would define the conformation of the substrate and/or a fluorinated carbocation intermediate. Our previous study suggested that the fluorocyclization 
of alkenes proceeds via the formation of a fluoro-carbocation intermediate $[40,41]$. If this is the case, hydrogen bond interaction of the cationic intermediate with the catalyst seems essential, because the intramolecular cyclization step is an enantio-determining step. Herein, we report our effort to develop the enantioselective 5-exo-fluorocyclization of ene-oximes to provide fluorinated isoxazolines (Scheme 1b).

(a) Our previous work

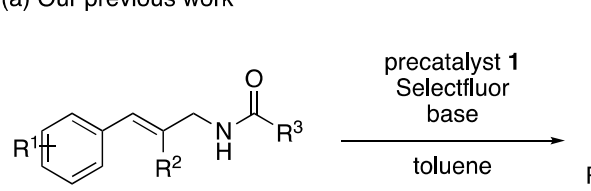

(b) This work
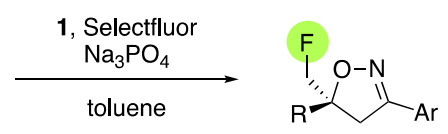

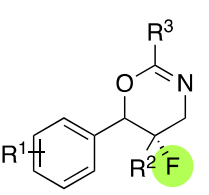

up to $99 \%$ ee<smiles>[R]C(=C)C/C([Al])=N/O</smiles>

Scheme 1. Fluorofunctionalizations of alkenes with linked-binaphthyl dicarboxylic acid 1 and Selectfluor: (a) Asymmetric 6-endo-type fluorocyclization of allylic amides; (b) Asymmetric 5-exo-fluorocyclization of ene-oximes.

\section{Results and Discussion}

Ene-oxime 2a [21] was chosen as a test substrate to optimize the reaction conditions (Table 1). At first, the reaction was carried out with 1 under the previous cyclization conditions [40] and the desired product, 3a, was obtained with 58\% ee (entry 1 ). In this reaction, undesired byproducts were simultaneously observed by ${ }^{1} \mathrm{H}$ NMR analysis of the crude mixture. Although these byproducts could not be purified at this point, ${ }^{1} \mathrm{H}$ and ${ }^{19} \mathrm{~F}$ NMR analyses suggested that the byproducts were deprotonative fluorination products $4 \mathbf{a}-\mathbf{6 a}$. The enantioselectivity observed in chlorobenzene and benzene was almost the same with that in toluene, but the reaction rate became somewhat slower (entries 1-3). Use of $\mathrm{CH}_{2} \mathrm{Cl}_{2}$ and THF resulted in low chemical yield (entries 4 and 5). While $\mathrm{Na}_{2} \mathrm{SO}_{4}$ had a positive effect in improving the chemical yield in our previous case [40,41], it did not affect the reaction efficiency in the present reaction (entries 1 and 6). Among bases tested, $\mathrm{Na}_{3} \mathrm{PO}_{4}$ was found to be the base of choice in terms of the chemical yield of the desired product 3a (entries 6-11). The enantioselectivity was almost similar (59\%-61\%), irrespective of the basicity and counter cation. Proton sponge provided a low yield and a low enantioselectivity under the described conditions (entry 11). As seen in entry 12 , the reaction rate became slower at $15^{\circ} \mathrm{C}$, but a better enantioselectivity was observed $(69 \%$ ee). In all cases, the yields based on the recovered starting material were around $65 \%$. Interestingly, the ee of $\mathbf{6 a}$ was as high as 77\% under the optimized conditions (entry 12), albeit in only $4 \%$ yield. It should be noted that phosphoric acid 7, which is a commonly used precursor of anionic phase transfer catalysts [33], did not promote the present reaction (entry 14), confirming the better performance of our dicarboxylate catalyst. 
Table 1. Optimization of the reaction conditions. ${ }^{1}$

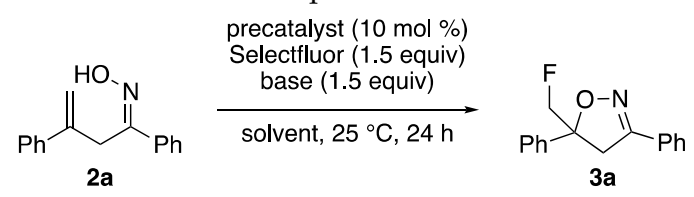

\begin{tabular}{|c|c|c|c|c|c|}
\hline \multirow[b]{2}{*}{ Entry } & etectable byprod & $\underbrace{\mathrm{F}}_{\mathbf{5 a}} \overbrace{P h}^{\mathrm{HO}}$ & $6 a$ & $\begin{array}{c}\mathrm{Ar} \\
2,4,6-\operatorname{tri}(i-\mathrm{Pr}) \mathrm{C}\end{array}$ & \\
\hline & Precatalyst & Solvent & Base & Yield (\%) ${ }^{2}$ & $\operatorname{Ee}(\%)^{3}$ \\
\hline $1^{4}$ & 1 & toluene & $\mathrm{Na}_{3} \mathrm{PO}_{4}$ & 39 & 58 \\
\hline $2^{4}$ & 1 & chlorobenzene & $\mathrm{Na}_{3} \mathrm{PO}_{4}$ & 26 & 58 \\
\hline $3^{4}$ & 1 & benzene & $\mathrm{Na}_{3} \mathrm{PO}_{4}$ & 30 & 57 \\
\hline $4^{4}$ & 1 & $\mathrm{CH}_{2} \mathrm{Cl}_{2}$ & $\mathrm{Na}_{3} \mathrm{PO}_{4}$ & 11 & - \\
\hline $5^{4}$ & 1 & THF & $\mathrm{Na}_{3} \mathrm{PO}_{4}$ & 3 & - \\
\hline 6 & 1 & toluene & $\mathrm{Na}_{3} \mathrm{PO}_{4}$ & 42 & 59 \\
\hline 7 & 1 & toluene & $\mathrm{Na}_{2} \mathrm{HPO}_{4}$ & 19 & 61 \\
\hline 8 & 1 & toluene & $\mathrm{Na}_{2} \mathrm{CO}_{3}$ & 35 & 61 \\
\hline 9 & 1 & toluene & $\mathrm{K}_{3} \mathrm{PO}_{4}$ & 24 & 59 \\
\hline 10 & 1 & toluene & $\mathrm{K}_{2} \mathrm{CO}_{3}$ & 3 & - \\
\hline 11 & 1 & toluene & proton sponge & 17 & 7 \\
\hline $12^{5}$ & 1 & toluene & $\mathrm{Na}_{3} \mathrm{PO}_{4}$ & $63(58)^{6}$ & 69 \\
\hline $13^{5}$ & 1 & toluene & $\mathrm{Na}_{2} \mathrm{CO}_{3}$ & $62(58)^{6}$ & 65 \\
\hline $14^{5}$ & 7 & toluene & $\mathrm{Na}_{3} \mathrm{PO}_{4}$ & 2 & - \\
\hline
\end{tabular}

${ }^{1}$ The reactions were carried out with $2 \mathrm{a}(0.1 \mathrm{mmol})$, precatalyst $(10 \mathrm{~mol} \%)$, Selectfluor $(1.5$ equiv), and base (1.5 equiv) at $25{ }^{\circ} \mathrm{C}$, unless otherwise mentioned. ${ }^{2}$ The yields were determined by ${ }^{1} \mathrm{H}$ NMR analysis using 1,1,2,2-tetrabromoethane as an internal standard. ${ }^{3}$ The ee values were determined by HPLC analysis using a chiral stationary column. ${ }^{4}$ Run with $\mathrm{Na}_{2} \mathrm{SO}_{4} \cdot{ }^{5}$ Run at $15{ }^{\circ} \mathrm{C}$ for $72 \mathrm{~h} .{ }^{6}$ Isolated yield.

To determine the absolute stereochemistry of the major isomer, the fluorinated isoxazoline 3a was transformed to the corresponding iodinated isoxazoline $8 \mathbf{a}$ with $\mathrm{MgI}_{2}$ using a sealed tube at $80{ }^{\circ} \mathrm{C}$ [42] (Scheme 2). Although the conversion was modest, 8 a could be obtained without erosion of the ee value. The stereochemistry of the major isomer was determined to be $S$ by comparing the retention time of HPLC analysis with that reported in the literature [21]. This result indicates that the major isomer of the present fluorocyclization is $S$.<smiles>FCC1(c2ccccc2)CC(c2ccccc2)=NO1</smiles>

3a : $65 \%$ ee

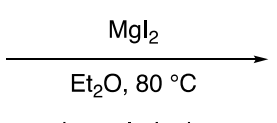

in sealed tube

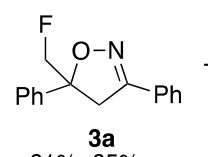

$61 \%, 65 \%$ ee

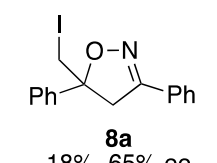

$18 \%, 65 \%$ ee

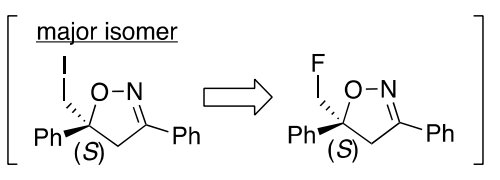

Scheme 2. Conversion of 3a to 8 a to determine the stereochemistry of the major product.

Having optimized the reaction conditions, other ene-oximes were transformed to the corresponding fluorinated isoxazolines (Figure 1). Reactions of para- and meta-methylated substrates proceeded to give the corresponding isoxazoline products with good ee values $(\mathbf{3 b}, \mathbf{3 c})$. However, ortho-substituent retarded the reaction completely, probably due to the steric repulsion (3d). meta-Chlorinated and para-fluorinated isoxazolines were formed with $77 \%$ ee and $73 \%$ ee, respectively (3e and $3 \mathbf{f}$ ). An ene-oxime bearing a cyclohexyl group was less reactive for the fluorocyclization and $3 \mathrm{~g}$ was obtained in only $12 \%$ yield, even at room temperature. The chemical structure on the oxime side did not have a significant impact on the enantioselectivity ( $3 \mathbf{h}$ and $3 \mathbf{i}$ ). To our delight, a substrate having a thiophen group provided $3 \mathbf{j}$ in $69 \%$ with $84 \%$ ee. We additionally performed the SDE 
(self-disproportionation of enantiomer) test by achiral column chromatography [43]. The difference between the first and the last fractions was less than 1\% ee, suggesting that SDE did not occur. As usual, all fractions were collected after the chromatographic purification.

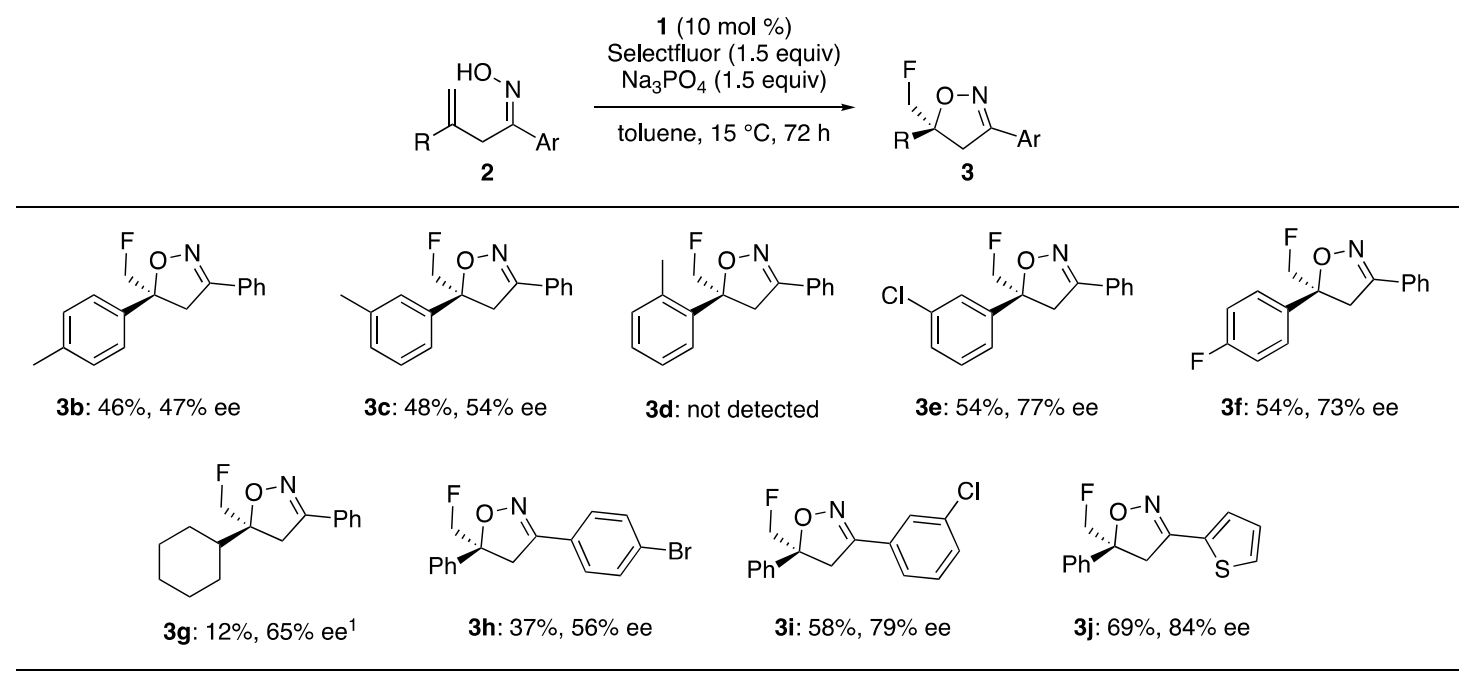

${ }^{1}$ Run at room temperature.

Figure 1. Fluorocyclization of ene-oximes.

To confirm the importance of hydrogen bond interaction between the oxime and the dianionic catalyst 1, the following control experiment was carried out. Thus, when $O$-methylated compound 9 was subjected to the described reaction conditions, no reaction occurred at all and the starting material 9 was just recovered (Scheme 3). Since even deprotonated products were not formed, it is likely that the hydrogen bond interaction has an important role in accelerating the fluorination step, in addition to the enantioselectivity control.
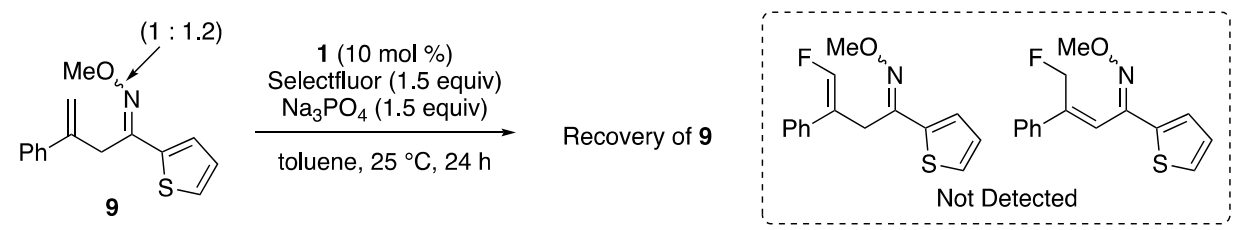

Scheme 3. Reaction of $O$-methylated compound.

\section{Materials and Methods}

\subsection{General Information}

${ }^{1} \mathrm{H}$ and ${ }^{19} \mathrm{~F}$ NMR spectra were measured on a JEOL ECX-500 spectrometer at 500 and $470 \mathrm{MHz}$, respectively. ${ }^{13} \mathrm{C}$ NMR spectra were recorded on a JEOL JNM-ECX-500 spectrometer at $125 \mathrm{MHz}$. Chemical shifts were reported in parts per million $(\mathrm{ppm})$ downfield from TMS $(\delta=0)$ for ${ }^{1} \mathrm{H}$ NMR. For ${ }^{13} \mathrm{C}$ NMR, chemical shifts were reported in the scale relative to $\mathrm{CDCl}_{3}$. For ${ }^{19} \mathrm{~F} \mathrm{NMR}$, chemical shifts were reported in a scale relative to $\mathrm{CFCl}_{3}$ external standard ( $\left.\delta=0 \mathrm{ppm}\right)$. Column chromatography was performed with silica gel N-60 (40-100 $\mu \mathrm{m})$ purchased from Kanto Chemical Co., Inc. TLC analysis was performed on Silica gel $60 \mathrm{~F}_{254}$-coated glass plates (Merck). Visualization was accomplished by means of ultraviolet (UV) irradiation at $254 \mathrm{~nm}$ or by spraying an ethanol solution of 12-molybdo(VI)phosphoric acid as a developing agent.

Dehydrated dichloromethane $\left(\mathrm{CH}_{2} \mathrm{Cl}_{2}\right)$ and toluene were purchased from Wako Pure Chemical Industries, Ltd. Dehydrated tetrahydrofuran (THF), diethyl ether $\left(\mathrm{Et}_{2} \mathrm{O}\right)$, and benzene were purchased 
from Kanto Chemical Co., Inc. Selectfluor was purchased from Aldrich. Other reagents were purified by usual methods.

The substrates were synthesized according to the literature [21]. The catalysts were synthesized according to the literature [40]. The ${ }^{1} \mathrm{H},{ }^{13} \mathrm{C}$ and ${ }^{19} \mathrm{~F}$ NMR spectra and HPLC data of compounds 3 are available in the Supplementary Material.

\subsection{Asymmetric Fluorocyclization of Ene-Oximes}

To a solution of $2 \mathrm{a}(23.7 \mathrm{mg}, 0.1 \mathrm{mmol}), \mathbf{1}(9.7 \mathrm{mg}, 10 \mathrm{~mol} \%)$, and $\mathrm{Na}_{3} \mathrm{PO}_{4}(24.6 \mathrm{mg}, 1.5$ equiv) in toluene $(1 \mathrm{~mL})$, was added Selectfluor $\left(53.1 \mathrm{mg}, 1.5\right.$ equiv) at $15^{\circ} \mathrm{C}$ under Ar atmosphere. After stirring for $72 \mathrm{~h}$ at $15^{\circ} \mathrm{C}$, the reaction mixture was diluted with EtOAc and filtrated through a pad of Celite. The filtrate was concentrated under reduced pressure. The resulting residue was purified by column chromatography on silica gel ( $n$-hexane/ethyl acetate $=20 / 1 \rightarrow 15 / 1 \rightarrow 10 / 1)$ to provide 3 a as a colorless solid (14.8 $\mathrm{mg}, 58 \%)$. The ee value was determined by chiral HPLC analysis.

5-(Fluoromethyl)-3,5-diphenylisoxazoline (3a). Colorless solid $(58 \%, 14.8 \mathrm{mg}) .[\alpha]^{27} \mathrm{D}=29.7$ $\left(\mathrm{c}=0.47, \mathrm{CHCl}_{3}\right)$. The ee value $(69 \%$ ee) was determined by HPLC analysis using a chiralpack IG-3 $(n$-hexane $/ i-\mathrm{PrOH}=80: 20$, flow rate $=1.0 \mathrm{~mL} / \mathrm{min}, \mathrm{t}($ minor $)=11.4 \mathrm{~min}, \mathrm{t}$ (major) $=20.6 \mathrm{~min}) .{ }^{1} \mathrm{H} \mathrm{NMR}$ $\left(500 \mathrm{MHz}, \mathrm{CDCl}_{3}\right) \delta=7.69-7.67(\mathrm{~m}, 2 \mathrm{H}), 7.53-7.51(\mathrm{~m}, 2 \mathrm{H}), 7.43-7.38(\mathrm{~m}, 5 \mathrm{H}), 7.36-7.33(\mathrm{~m}, 1 \mathrm{H}), 4.64$ $(\mathrm{dd}, J=9.7,47.5 \mathrm{~Hz}, 1 \mathrm{H}), 4.60(\mathrm{dd}, J=9.7,47.5 \mathrm{~Hz}, 1 \mathrm{H}), 3.89(\mathrm{~d}, J=16.6 \mathrm{~Hz}, 1 \mathrm{H}), 3.53(\mathrm{dd}, J=2.3,16.6$ $\mathrm{Hz}, 1 \mathrm{H}) .{ }^{13} \mathrm{C} \mathrm{NMR}\left(125 \mathrm{MHz}, \mathrm{CDCl}_{3}\right) \delta=156.2,140.1(\mathrm{~d}, J=3.6 \mathrm{~Hz}), 130.3,129.3,128.7,128.7,128.4$, $126.7,125.5,88.9(\mathrm{~d}, J=18.0 \mathrm{~Hz}), 85.5(\mathrm{~d}, J=183.5 \mathrm{~Hz}), 42.9(\mathrm{~d}, J=3.6 \mathrm{~Hz}) .{ }^{19} \mathrm{~F} \mathrm{NMR}\left(470 \mathrm{MHz}, \mathrm{CDCl}_{3}\right)$ $\delta=-221.3(\mathrm{t}, J=47.5 \mathrm{~Hz}, 1 \mathrm{~F})$. IR (neat): 2941, 1599, 1447, 1362, 1020, 980, $912 \mathrm{~cm}^{-1}$. HRMS (ESI) m/z calcd. for $\mathrm{C}_{16} \mathrm{H}_{15} \mathrm{FNO}\left[\mathrm{M}+\mathrm{H}^{+}\right]$: 256.1132, found: 256.1131 .

5-(Fluoromethyl)-3-phenyl-5-(p-tolyl)isoxazoline (3b). Colorless solid $(46 \%, 12.7 \mathrm{mg}) \cdot[\alpha]^{29} \mathrm{D}=$ $31.8\left(\mathrm{c}=0.38, \mathrm{CHCl}_{3}\right)$. The ee value $(47 \%$ ee) was determined by HPLC analysis using a chiralpack IG-3 (n-hexane $/ i-\mathrm{PrOH}=80: 20$, flow rate $=1.0 \mathrm{~mL} / \mathrm{min}, \mathrm{t}$ (minor) $=13.4 \mathrm{~min}, \mathrm{t}$ (major) $=23.5 \mathrm{~min}$ ). ${ }^{1} \mathrm{H} \mathrm{NMR}$ $\left(500 \mathrm{MHz}, \mathrm{CDCl}_{3}\right) \delta=7.67-7.66(\mathrm{~m}, 2 \mathrm{H}), 7.42-7.37(\mathrm{~m}, 5 \mathrm{H}), 7.21(\mathrm{~d}, J=8.0 \mathrm{~Hz}, 2 \mathrm{H}), 4.61(\mathrm{dd}, J=10.0$, $47.4 \mathrm{~Hz}, 1 \mathrm{H}), 4.57(\mathrm{dd}, J=10.0,47.4 \mathrm{~Hz}, 1 \mathrm{H}) 3.86(\mathrm{~d}, J=16.6 \mathrm{~Hz}, 1 \mathrm{H}), 3.51(\mathrm{dd}, J=1.7,16.6 \mathrm{~Hz}, 1 \mathrm{H})$, $2.35(\mathrm{~s}, 3 \mathrm{H}) .{ }^{13} \mathrm{C}$ NMR $\left(125 \mathrm{MHz}, \mathrm{CDCl}_{3}\right) \delta=156.2,138.2,137.1(\mathrm{~d}, J=3.6 \mathrm{~Hz}), 130.2,129.4,129.3,128.7$, 126.6, $125.4,88.9(\mathrm{~d}, J=18.0 \mathrm{~Hz}), 85.5(\mathrm{~d}, J=183.5 \mathrm{~Hz}), 42.8(\mathrm{~d}, J=3.6 \mathrm{~Hz}), 21.0 .{ }^{19} \mathrm{~F} \mathrm{NMR}(470 \mathrm{MHz}$, $\left.\mathrm{CDCl}_{3}\right) \delta=-220.9(\mathrm{t}, J=47.4 \mathrm{~Hz}, 1 \mathrm{~F})$. IR (neat): 2922, 1570, 1514, 1447, 1358, 978, $906 \mathrm{~cm}^{-1}$. HRMS (ESI) $\mathrm{m} / \mathrm{z}$ calcd. for $\mathrm{C}_{17} \mathrm{H}_{17} \mathrm{FNO}$ [M $+\mathrm{H}^{+}$]: 270.1289, found: 270.1287 .

5-(Fluoromethyl)-3-phenyl-5-(m-tolyl)isoxazoline (3c). Colorless solid $(48 \%, 12.9 \mathrm{mg}) \cdot[\alpha]^{28}{ }_{\mathrm{D}}=$ $21.4\left(\mathrm{c}=0.50, \mathrm{CHCl}_{3}\right)$. The ee value ( $54 \%$ ee) was determined by HPLC analysis using a chiralpack IG-3 ( $n$-hexane $/ i-\mathrm{PrOH}=80: 20$, flow rate $=1.0 \mathrm{~mL} / \mathrm{min}, \mathrm{t}$ (minor) $=8.7 \mathrm{~min}, \mathrm{t}$ (major) $=12.4 \mathrm{~min}) .{ }^{1} \mathrm{H} \mathrm{NMR}$ $\left(500 \mathrm{MHz}, \mathrm{CDCl}_{3}\right) \delta=7.69-7.68(\mathrm{~m}, 2 \mathrm{H}), 7.41-7.39(\mathrm{~m}, 3 \mathrm{H}), 7.35(\mathrm{~s}, 1 \mathrm{H}), 7.30-7.29(\mathrm{~m}, 2 \mathrm{H}), 7.17-7.15(\mathrm{~m}$, $1 \mathrm{H}), 4.63(\mathrm{dd}, J=10.0,47.6 \mathrm{~Hz}, 1 \mathrm{H}), 4.60(\mathrm{dd}, J=10.0,47.6 \mathrm{~Hz}, 1 \mathrm{H}), 3.88(\mathrm{~d}, J=16.6 \mathrm{~Hz}, 1 \mathrm{H}), 3.53(\mathrm{dd}$, $J=2.3,16.6 \mathrm{~Hz}, 1 \mathrm{H}), 2.39(\mathrm{~s}, 3 \mathrm{H}) .{ }^{13} \mathrm{C} \mathrm{NMR}\left(125 \mathrm{MHz}, \mathrm{CDCl}_{3}\right) \delta=156.2,140.0(\mathrm{~d}, J=3.6 \mathrm{~Hz}), 138.5$, $130.2,129.3,129.1,128.7,128.6,126.6,126.1,122.5,88.9(\mathrm{~d}, J=18.0 \mathrm{~Hz}), 85.6(\mathrm{~d}, J=183.5 \mathrm{~Hz}), 42.8(\mathrm{~d}$, $J=3.6 \mathrm{~Hz}), 21.5 .{ }^{19} \mathrm{~F}$ NMR $\left(470 \mathrm{MHz}, \mathrm{CDCl}_{3}\right) \delta=-221.1(\mathrm{t}, J=47.6 \mathrm{~Hz}, 1 \mathrm{~F})$. IR (neat): 2955, 1600, 1487, 1447, 1364, 1011, $922 \mathrm{~cm}^{-1}$. HRMS (ESI) m/z calcd. for $\mathrm{C}_{17} \mathrm{H}_{17} \mathrm{FNO}\left[\mathrm{M}+\mathrm{H}^{+}\right]: 270.1289$, found: 270.1286 .

5-(3-Chlorophenyl)-5-(fluoromethyl)-3-phenylisoxazoline (3e). Colorless oil (54\%, $15.6 \mathrm{mg}) \cdot[\alpha]^{27} \mathrm{D}$ $=33.3\left(\mathrm{c}=0.19, \mathrm{CHCl}_{3}\right)$. The ee value $(77 \%$ ee) was determined by HPLC analysis using a chiralpack IG-3 ( $n$-hexane $/ \mathrm{i}-\mathrm{PrOH}=80: 20$, flow rate $=1.0 \mathrm{~mL} / \mathrm{min}, \mathrm{t}$ (minor) $=8.8 \mathrm{~min}, \mathrm{t}($ major $)=10.2 \mathrm{~min}$ ). ${ }^{1} \mathrm{H}$ NMR $\left(500 \mathrm{MHz}, \mathrm{CDCl}_{3}\right) \delta=7.68-7.66(\mathrm{~m}, 2 \mathrm{H}), 7.53(\mathrm{~s}, 1 \mathrm{H}), 7.41-7.39(\mathrm{~m}, 4 \mathrm{H}), 7.36-7.31(\mathrm{~m}, 2 \mathrm{H})$, $4.62(\mathrm{dd}, J=10.0,47.0 \mathrm{~Hz}, 1 \mathrm{H}), 4.56(\mathrm{dd}, J=10.0,47.0 \mathrm{~Hz}, 1 \mathrm{H}), 3.88(\mathrm{~d}, J=16.6 \mathrm{~Hz}, 1 \mathrm{H}), 3.50(\mathrm{dd}, J=2.3$, $16.6 \mathrm{~Hz}, 1 \mathrm{H}) .{ }^{13} \mathrm{C}$ NMR $\left(125 \mathrm{MHz}, \mathrm{CDCl}_{3}\right) \delta=156.2,142.3(\mathrm{~d}, J=2.4 \mathrm{~Hz}), 134.8,130.4,130.1,128.9$, $128.8,128.6,126.7,125.9,123.7,88.3(\mathrm{~d}, J=19.2 \mathrm{~Hz}), 85.1(\mathrm{~d}, J=183.5 \mathrm{~Hz}), 43.1(\mathrm{~d}, J=3.6 \mathrm{~Hz}) .{ }^{19} \mathrm{~F}$ NMR $\left(470 \mathrm{MHz}, \mathrm{CDCl}_{3}\right) \delta=-221.6(\mathrm{t}, J=47.0 \mathrm{~Hz}, 1 \mathrm{~F})$. IR (neat): 2940, 1597, 1572, 1447, 1358, 1034, 995, $910 \mathrm{~cm}^{-1}$. HRMS (ESI) $\mathrm{m} / \mathrm{z}$ calcd. for $\mathrm{C}_{16} \mathrm{H}_{14} \mathrm{ClFNO}$ [M $+\mathrm{H}^{+}$]: 290.0742, found: 290.0743 . 
5-(Fluoromethyl)-5-(4-fluorophenyl)-3-phenylisoxazoline (3f). Colorless solid (54\%, $14.8 \mathrm{mg})$. $[\alpha]^{28} \mathrm{D}=27.6\left(\mathrm{c}=0.40, \mathrm{CHCl}_{3}\right)$. The ee value $(73 \%$ ee) was determined by HPLC analysis using a chiralpack IG-3 ( $n$-hexane $/ \mathrm{i}-\mathrm{PrOH}=80: 20$, flow rate $=1.0 \mathrm{~mL} / \mathrm{min}, \mathrm{t}($ minor $)=10.1 \mathrm{~min}, \mathrm{t}($ major $)=17.1$ min). ${ }^{1} \mathrm{H}$ NMR (500 MHz, $\left.\mathrm{CDCl}_{3}\right) \delta=7.68-7.66(\mathrm{~m}, 2 \mathrm{H}), 7.51-7.48(\mathrm{~m}, 2 \mathrm{H}), 7.42-7.38(\mathrm{~m}, 3 \mathrm{H}), 7.11-7.08$ $(\mathrm{m}, 2 \mathrm{H}), 4.61(\mathrm{dd}, J=9.7,47.5 \mathrm{~Hz}, 1 \mathrm{H}), 4.57(\mathrm{dd}, J=9.7,47.5 \mathrm{~Hz}, 1 \mathrm{H}), 3.88(\mathrm{~d}, J=16.6 \mathrm{~Hz}, 1 \mathrm{H}), 3.49(\mathrm{dd}$, $J=2.3,16.6 \mathrm{~Hz}, 1 \mathrm{H}) .{ }^{13} \mathrm{C} \mathrm{NMR}\left(125 \mathrm{MHz}, \mathrm{CDCl}_{3}\right) \delta=162.6(\mathrm{~d}, J=247.1 \mathrm{~Hz}), 156.2,136.0(\mathrm{t}, J=3.6$ $\mathrm{Hz}), 130.4,129.1,128.8,127.4(\mathrm{~d}, J=8.4 \mathrm{~Hz}), 126.7,115.7(\mathrm{~d}, J=21.6 \mathrm{~Hz}), 88.4(\mathrm{~d}, J=19.2 \mathrm{~Hz}), 85.3(\mathrm{~d}$, $J=183.5 \mathrm{~Hz}), 43.1(\mathrm{~d}, J=3.6 \mathrm{~Hz}) .{ }^{19} \mathrm{~F}$ NMR $\left(470 \mathrm{MHz}, \mathrm{CDCl}_{3}\right) \delta=-113.5--113.6(\mathrm{~m}, 1 \mathrm{~F}),-221.1(\mathrm{t}$, $J=47.5 \mathrm{~Hz}, 1 \mathrm{~F})$. IR (neat): 1601, 1508, 1447, 1360, 1221, 1159, 1028, $918 \mathrm{~cm}^{-1}$. HRMS (ESI) m/z calcd. for $\mathrm{C}_{16} \mathrm{H}_{14} \mathrm{~F}_{2} \mathrm{NO}\left[\mathrm{M}+\mathrm{H}^{+}\right]$: 274.1038, found: 274.1034 .

5-Cyclohexyl-5-(fluoromethyl)-3-phenylisoxazoline (3g). Colorless oil $(12 \%, 3.1 \mathrm{mg}) .[\alpha]^{29}{ }_{\mathrm{D}}=$ $45.8\left(\mathrm{c}=0.34, \mathrm{CHCl}_{3}\right)$. The ee value $(65 \%$ ee) was determined by HPLC analysis using a chiralpack IG-3 ( $n$-hexane $/ i$-PrOH $=80: 20$, flow rate $=1.0 \mathrm{~mL} / \mathrm{min}, \mathrm{t}$ (minor) $=8.2 \mathrm{~min}, \mathrm{t}($ minor $)=9.2 \mathrm{~min}) .{ }^{1} \mathrm{H}$ $\operatorname{NMR}\left(500 \mathrm{MHz}, \mathrm{CDCl}_{3}\right) \delta=7.68-7.66(\mathrm{~m}, 2 \mathrm{H}), 7.41-7.39(\mathrm{~m}, 3 \mathrm{H}), 4.53(\mathrm{dd}, J=9.7,47.0 \mathrm{~Hz}, 1 \mathrm{H}), 4.49$ $(\mathrm{dd}, J=9.7,47.0 \mathrm{~Hz}, 1 \mathrm{H}), 3.22(\mathrm{~d}, J=1.7 \mathrm{~Hz}, 2 \mathrm{H}), 1.82-1.78(\mathrm{~m}, 5 \mathrm{H}), 1.71-1.69(\mathrm{~m}, 1 \mathrm{H}), 1.32-1.05(\mathrm{~m}$, 5H). ${ }^{13} \mathrm{C} \mathrm{NMR}\left(125 \mathrm{MHz}, \mathrm{CDCl}_{3}\right) \delta=155.7,130.0,129.6,128.7,126.5,90.2(\mathrm{~d}, J=16.8 \mathrm{~Hz}), 84.2(\mathrm{~d}$, $J=177.5 \mathrm{~Hz}), 42.6(\mathrm{~d}, J=2.4 \mathrm{~Hz}), 37.9(\mathrm{~d}, J=6.0 \mathrm{~Hz}), 27.2,26.5,26.2,26.1,26.1 .{ }^{19} \mathrm{~F} \mathrm{NMR}(470 \mathrm{MHz}$, $\left.\mathrm{CDCl}_{3}\right) \delta=-229.6(\mathrm{t}, J=47.0 \mathrm{~Hz}, 1 \mathrm{~F})$. IR (neat): 2922, 1595, 1447, 1360, 1009, $926 \mathrm{~cm}^{-1}$. HRMS (ESI) $\mathrm{m} / \mathrm{z}$ calcd. for $\mathrm{C}_{16} \mathrm{H}_{21} \mathrm{FNO}\left[\mathrm{M}+\mathrm{H}^{+}\right]$: 262.1602, found: 262.1609 .

3-(4-Bromophenyl)-5-(fluoromethyl)-5-phenylisoxazoline (3h). Colorless solid (37\%, $12.4 \mathrm{mg})$. $[\alpha]_{\mathrm{D}}^{28}=22.5\left(\mathrm{c}=0.39, \mathrm{CHCl}_{3}\right)$. The ee value $(56 \%$ ee) was determined by HPLC analysis using a chiralpack IG-3 ( $n$-hexane $/ \mathrm{i}-\mathrm{PrOH}=80: 20$, flow rate $=1.0 \mathrm{~mL} / \mathrm{min}, \mathrm{t}$ (minor) $=12.9 \mathrm{~min}, \mathrm{t}($ major $)=20.4$ min). ${ }^{1} \mathrm{H}$ NMR (500 MHz, $\left.\mathrm{CDCl}_{3}\right) \delta=7.55-7.49(\mathrm{~m}, 6 \mathrm{H}), 7.43-7.40(\mathrm{~m}, 2 \mathrm{H}), 7.36-7.33(\mathrm{~m}, 1 \mathrm{H}), 4.63(\mathrm{dd}$, $J=10.3,47.1 \mathrm{~Hz}, 1 \mathrm{H}), 4.59(\mathrm{dd}, J=10.3,47.1 \mathrm{~Hz}, 1 \mathrm{H}), 3.86(\mathrm{~d}, J=16.6 \mathrm{~Hz}, 1 \mathrm{H}), 3.50(\mathrm{dd}, J=2.3,16.6 \mathrm{~Hz}$, $1 \mathrm{H}) .{ }^{13} \mathrm{C}$ NMR $\left(125 \mathrm{MHz}, \mathrm{CDCl}_{3}\right) \delta=155.3,139.8(\mathrm{~d}, J=4.8 \mathrm{~Hz}), 131.9,128.8,128.5,128.2,128.1,125.4$, $124.5,89.3(\mathrm{~d}, J=18.0 \mathrm{~Hz}), 85.5(\mathrm{~d}, J=184.7 \mathrm{~Hz}), 42.6(\mathrm{~d}, J=3.6 \mathrm{~Hz}) .{ }^{19} \mathrm{~F} \mathrm{NMR}\left(470 \mathrm{MHz}, \mathrm{CDCl}_{3}\right) \delta=$ -221.2 (t, $J=47.1 \mathrm{~Hz}, 1 \mathrm{~F})$. IR (neat): 2951, 1589, 1489, 1447, 1396, 1356, 1246, $989 \mathrm{~cm}^{-1}$. HRMS (ESI) m/z calcd. for $\mathrm{C}_{16} \mathrm{H}_{14} \mathrm{BrFNO}\left[\mathrm{M}+\mathrm{H}^{+}\right.$]: 334.0237, found: 334.0237 .

3-(3-Chlorophenyl)-5-(fluoromethyl)-5-phenylisoxazoline (3i). Colorless oil (58\%, $16.8 \mathrm{mg}) \cdot[\alpha]^{28} \mathrm{D}$ $=43.5\left(\mathrm{c}=0.30, \mathrm{CHCl}_{3}\right)$. The ee value $(79 \%$ ee) was determined by HPLC analysis using a chiralpack IG-3 (n-hexane $/ i$-PrOH $=80: 20$, flow rate $=1.0 \mathrm{~mL} / \mathrm{min}, \mathrm{t}$ (minor) $=8.1 \mathrm{~min}, \mathrm{t}$ (major) $=9.7 \mathrm{~min}) .{ }^{1} \mathrm{H}$ $\operatorname{NMR}\left(500 \mathrm{MHz}, \mathrm{CDCl}_{3}\right) \delta=7.66(\mathrm{~s}, 1 \mathrm{H}), 7.57(\mathrm{~d}, J=7.5 \mathrm{~Hz}, 1 \mathrm{H}), 7.50(\mathrm{~d}, J=8.0 \mathrm{~Hz}, 2 \mathrm{H}), 7.43-7.40(\mathrm{~m}$, $2 \mathrm{H}), 7.38-7.31(\mathrm{~m}, 3 \mathrm{H}), 4.64(\mathrm{dd}, J=10.3,47.1 \mathrm{~Hz}, 1 \mathrm{H}), 4.60(\mathrm{dd}, J=10.3,47.1 \mathrm{~Hz}, 1 \mathrm{H}), 3.87(\mathrm{~d}, J=16.6$ $\mathrm{Hz}, 1 \mathrm{H}), 3.51(\mathrm{dd}, J=2.3,16.6 \mathrm{~Hz}, 1 \mathrm{H}) .{ }^{13} \mathrm{C} \mathrm{NMR}\left(125 \mathrm{MHz}, \mathrm{CDCl}_{3}\right) \delta=155.2,139.7(\mathrm{~d}, J=3.6 \mathrm{~Hz})$, 134.7, 131.0, 130.2, 130.0, 128.8, 128.5, 126.7, 125.4, 124.7, 89.4 (d, J = 18.0 Hz), $85.5(\mathrm{~d}, J=184.7 \mathrm{~Hz})$, $42.5(\mathrm{~d}, J=3.6 \mathrm{~Hz}) .{ }^{19} \mathrm{~F} \mathrm{NMR}\left(470 \mathrm{MHz}, \mathrm{CDCl}_{3}\right) \delta=-221.2(\mathrm{t}, J=47.1 \mathrm{~Hz}) . \mathrm{IR}$ (neat): 3063, 2947, 1595, 1560, 1429, 1344, 1028, $918 \mathrm{~cm}^{-1}$. HRMS (ESI) m/z calcd. for $\mathrm{C}_{16} \mathrm{H}_{14} \mathrm{ClFNO}\left[\mathrm{M}+\mathrm{H}^{+}\right]$: 290.0742, found: 290.0724 .

5-(fluoromethyl)-5-phenyl-3-(3-thiophen-2-yl)isoxazoline (3j). Colorless solid (69\%, $18.0 \mathrm{mg})$. $[\alpha]^{28}=14.3\left(\mathrm{c}=0.25, \mathrm{CHCl}_{3}\right)$. The ee value $(84 \%$ ee) was determined by HPLC analysis using a chiralpack IG-3 ( $n$-hexane $/ \mathrm{i}-\mathrm{PrOH}=80: 20$, flow rate $=1.0 \mathrm{~mL} / \mathrm{min}, \mathrm{t}($ minor $)=11.8 \mathrm{~min}, \mathrm{t}($ major $)=21.6$ min). ${ }^{1} \mathrm{H}$ NMR $\left(500 \mathrm{MHz}, \mathrm{CDCl}_{3}\right) \delta=7.52-7.50(\mathrm{~m}, 2 \mathrm{H}), 7.43-7.38(\mathrm{~m}, 3 \mathrm{H}), 7.36-7.33(\mathrm{~m}, 1 \mathrm{H}), 7.21-7.20$ $(\mathrm{m}, 1 \mathrm{H}), 7.06-7.04(\mathrm{~m}, 1 \mathrm{H}), 4.63(\mathrm{dd}, J=10.3,47.5 \mathrm{~Hz}, 1 \mathrm{H}), 4.59(\mathrm{dd}, J=10.3,47.5 \mathrm{~Hz}, 1 \mathrm{H}), 3.90(\mathrm{~d}$, $J=16.2 \mathrm{~Hz}, 1 \mathrm{H}), 3.53(\mathrm{dd}, J=2.6,16.2 \mathrm{~Hz}, 1 \mathrm{H}) .{ }^{13} \mathrm{C} \mathrm{NMR}\left(125 \mathrm{MHz}, \mathrm{CDCl}_{3}\right) \delta=152.0,139.8(\mathrm{~d}, J=$ $3.6 \mathrm{~Hz}), 131.7,128.8,128.5,128.5,128.4,127.3,125.5,89.1(\mathrm{~d}, J=18.1 \mathrm{~Hz}), 85.3(\mathrm{~d}, J=183.5 \mathrm{~Hz}), 43.6$ $(\mathrm{d}, J=3.6 \mathrm{~Hz}) .{ }^{19} \mathrm{~F} \mathrm{NMR}\left(470 \mathrm{MHz}, \mathrm{CDCl}_{3}\right) \delta=-221.1(\mathrm{t}, J=47.5 \mathrm{~Hz})$. IR (neat): 3084, 2943, 1600, 1491, 1437, 1024, 982, $907 \mathrm{~cm}^{-1}$. HRMS (ESI) m/z calcd. for $\mathrm{C}_{14} \mathrm{H}_{13} \mathrm{FNOS}\left[\mathrm{M}+\mathrm{H}^{+}\right]$: 262.0696, found: 262.0695 . 


\section{Conclusions}

In this paper, we have demonstrated the enantioselective 5-exo-fluorocyclization of ene-oximes using the linked-binaphthyl dicarboxylic acid precatalyst 1 . The corresponding fluorinated isoxazolines were obtained with up to $84 \%$ ee, and the stereochemistry of the major isomers were determined to be $S$ after transformation to known isoxazoline 8a with an iodomethyl unit. A control experiment revealed that hydrogen bond interaction of the oxime group is extremely important for the reaction acceleration and the enantioselectivity control. Further applications of the present fluorinating system are underway in our laboratory.

Supplementary Materials: The following are available online, ${ }^{1} \mathrm{H},{ }^{13} \mathrm{C}$, and ${ }^{19} \mathrm{~F}$ NMR spectra of compounds 3 and HPLC data of compounds 3.

Author Contributions: H.E. and Y.H. conceived and directed this project. T.R., T.N., K.N., N.Y., and H.E. performed the experiments. H.E. and Y.H. wrote the paper.

Funding: This work was supported by a Grant-in-Aid for Scientific Research (Nos. 18K06585, and 19H03353) from JSPS, Basis for Supporting Innovative Drug Discovery and Life Science Research (BINDS) from Japan Agency for Medical Research and Development (AMED), The Uehara Memorial Foundation and Nagase Science and Technology Foundation.

Conflicts of Interest: The authors declare no conflict of interest.

\section{References}

1. Kaur, K.; Kumar, V.; Sharma, A.K.; Gupta, G.K. Isoxazoline containing natural products as anticancer agents: A review. Eur. J. Med. Chem. 2014, 77, 121-133. [CrossRef]

2. Schorderet-Weber, S.; Noack, S.; Selzer, P.M.; Kaminsky, R. Blocking transmission of vector-borne diseases. Int. J. Parasitol. Drugs Drug Resist. 2017, 7, 90-109. [CrossRef] [PubMed]

3. Kamal, A.; Reddy, J.S.; Ramaiah, M.J.; Dastagiri, D.; Bharathi, E.V.; Azhar, M.A.; Sultana, F.; Pushpavalli, S.; Pal-Bhadra, M.; Juvekar, A.; et al. Design, synthesis and biological evaluation of 3,5-diaryl-isoxazoline/isoxazole-pyrrolobenzodiazepine conjugates as potential anticancer agents. Eur. J. Med. Chem. 2010, 45, 3924-3937. [CrossRef]

4. Kamal, A.; Bharathi, E.V.; Reddy, J.S.; Ramaiah, M.J.; Dastagiri, D.; Reddy, M.K.; Viswanath, A.; Reddy, T.L.; Shaik, T.B.; Pushpavalli, S.; et al. Synthesis and biological evaluation of 3,5-diaryl isoxazoline/isoxazole linked 2,3-dihydroquinazolinone hybrids as anticancer agents. Eur. J. Med. Chem. 2011, 46, 691-703. [CrossRef] [PubMed]

5. Quan, M.L.; Ellis, C.D.; Liauw, A.Y.; Alexander, R.S.; Knabb, R.M.; Lam, G.; Wright, M.R.; Wong, P.C.; Wexler, R.R. Design and Synthesis of Isoxazoline Derivatives as Factor Xa Inhibitors. 2. J. Med. Chem. 1999, 42, 2760-2773. [CrossRef] [PubMed]

6. Ozoe, Y.; Asahi, M.; Ozoe, F.; Nakahira, K.; Mita, T. The antiparasitic isoxazoline A1443 is a potent blocker of insect ligand-gated chloride channels. Biochem. Biophys. Res. Commun. 2010, 391, 744-749. [CrossRef]

7. Gracia-Reynaga, P.; Zhao, C.; Sarpong, R.; Casida, J.E. New GABA/Glutamate Receptor Target for $\left[{ }^{3} \mathrm{H}\right]$ Isoxazoline Insecticide. Chem. Res. Toxicol. 2013, 26, 514-516. [CrossRef] [PubMed]

8. Shoop, W.L.; Hartline, E.J.; Gould, B.R.; Waddell, M.E.; McDowell, R.G.; Kinney, J.B.; Lahm, G.P.; Long, J.K.; $\mathrm{Xu}, \mathrm{M}$.; Wagerle, T.; et al. Discovery and mode of action of afoxolaner, a new isoxazoline parasiticide for dogs. Vet. Parasitol. 2014, 201, 179-189. [CrossRef]

9. Zhang, Y.-K.; Plattner, J.J; Easom, E.E.; Zhou, Y.; Akama, T.; Bu, W.; White, W.H.; Defauw, J.M.; Winkle, J.R.; Balko, T.W.; et al. Discovery of an orally bioavailable isoxazoline benzoxaborole (AN8030) as a long acting animal ectoparasiticide. Bioorg. Med. Chem. Lett. 2015, 25, 5589-5593. [CrossRef]

10. Madavu, K.; Rai, L. Heterocycles via Oxime Cycloadditions. In Synthesis of Heterocycles via Cycloadditions II; Springer: Berlin/Heidelberg, Germany, 2008; Volume 13, pp. 1-69.

11. Kawai, H.; Shibata, N. Attractive Trifluoromethylated Dihydroxazoles and Related Compounds under Organocatalysis. Chem. Rec. 2014, 14, 1024-1040. [CrossRef]

12. Kumer, V.; Kaur, K. Fluorinated isoxazolines and isoxazoles: A synthetic perspective. J. Fluor. Chem. 2015, 180, 55-97. [CrossRef] 
13. Yoshimura, A.; Zhdankin, V.V. Oxidative cyclizations of oximes using hypervalent iodine reagents. Arkivoc 2017, i, 99-116. [CrossRef]

14. Zhu, M.; Fun, W.; Guo, W.; Tian, Y.; Wang, Z.; Xu, C.; Ji, B. Visible-Light-Induced Radical Di- and Trifluoromethylation of $\beta, \gamma$-Unsaturated Oximes: Synthesis of Di- and Trifluoromethylated Isoxazolines. Eur. J. Org. Chem. 2019, 2019, 1614-1619. [CrossRef]

15. Wei, Q.; Chen, J.-R.; Hu, X.-Q.; Yang, X.-C.; Lu, B.; Xiao, W.-J. Photocatalytic Radical Trifluoromethylation/Cyclization Cascade: Synthesis of $\mathrm{CF}_{3}-$ Containing Pyrazolines and Isoxazolines. Org. Lett. 2015, 17, 4464-4467. [CrossRef]

16. Li, X.-T.; Lv, L.; Gu, Q.-S.; Liu, X.-Y. Copper-catalyzed radical oxytrifluoromethylation of alkenyl oximes at ambient temperature. Tetrahedron 2018, 74, 6041-6046. [CrossRef]

17. Li, X.-T.; Gu, Q.-S.; Dong, X.-Y.; Meng, X.; Liu, X.-Y. A Copper Catalyst with a Cinchona-Alkaloid-Based Sulfonamide Ligand for Asymmetric Radical Oxytrifluoromethylation of Alkenyl Oximes. Angew. Chem. Int. Ed. 2018, 57, 7668-7672. [CrossRef]

18. Zhu, M.-K.; Zhao, J.-F.; Loh, T.-P. Palladium-Catalyzed Oxime Assisted Intramolecular Dioxygenation of Alkenes with $1 \mathrm{~atm}$ of Air as the Sole Oxidant. J. Am. Chem. Soc. 2010, 132, 6284-6285. [CrossRef]

19. Yamamoto, D.; Oguro, T.; Tashiro, Y.; Soga, M.; Miyashita, K.; Aso, Y.; Makino, K. Manganese-Promoted Oxidative Cyclization of Unsaturated Oximes Using Molecular Oxygen in Air under Ambient Conditions. Eur. J. Org. Chem. 2016, 2016, 5216-5219. [CrossRef]

20. Ji, F.; Fan, Y.; Yang, R.; Yang, Y.; Yu, D.; Wang, M.; Li, Z. Regioselective synthesis of thiocyanate-containing isoxazolines via $\mathrm{Fe}(\mathrm{III}) / \mathrm{K}_{2} \mathrm{~S}_{2} \mathrm{O}_{8}$-mediated radical thiocyanation/cyclization cascade reaction of $\beta, \gamma$-unsaturated oximes. Asian J. Org. Chem. 2017, 6, 682-685. [CrossRef]

21. Tripathi, C.B.; Mukherjee, S. Catalytic Enantioselective Iodoetherification of Oximes. Angew. Chem. Int. Ed. 2013, 52, 8450-8453. [CrossRef]

22. Kirsch, P. Modern Fluoroorganic Chemistry: Synthesis, Reactivity, Applications, 2nd ed.; Wiley-VHC: Weinheim, Germany, 2013.

23. Ojima, I. Fluorine in Medicinal Chemistry and Chemical Biology; Wiley-Blackwell: Oxford, UK, 2009.

24. Gouverneur, V.; Müller, K. Fluorine in Pharmaceutical and Medicinal Chemistry: From Biophysical Aspects to Clinical Applications; Imperial College Press: London, UK, 2012.

25. Wang, J.; Sánchez-Roselló, M.; Aceña, J.L.; del Pozo, C.; Sorochinsky, A.E.; Fustero, S.; Soloshonok, V.A.; Liu, H. Fluorine in Pharmaceutical Industry: Fluorine-Containing Drugs Introduced to the Market in the Last Decade (2001-2011). Chem. Rev. 2014, 114, 2432-2506. [CrossRef] [PubMed]

26. Gillis, E.P.; Eastman, K.J.; Hill, M.D.; Donnelly, D.J.; Meanwell, N.A. Applications of Fluorine in Medicinal Chemistry. J. Med. Chem. 2015, 58, 8315-8359. [CrossRef] [PubMed]

27. Haranahalli, K.; Honda, T.; Ojima, I. Resent progress in the strategic incorporation of fluorine into medicinally active compounds. J. Fluor. Chem. 2019, 217, 29-40. [CrossRef] [PubMed]

28. Liang, T.; Neumann, C.N.; Ritter, T. Introduction of Fluorine and Fluorine-Containing Functional Groups. Angew. Chem. Int. Ed. 2013, 52, 8214-8264. [CrossRef] [PubMed]

29. Champagne, P.A.; Desroches, J.; Hamel, J.-D.; Vandamme, M.; Paquin, J.-F. Monofluorination of Organic Compounds: 10 Years of Innovation. Chem. Rev. 2015, 115, 9073-9174. [CrossRef] [PubMed]

30. Zhu, Y.; Han, J.; Wang, J.; Shibata, N.; Sodeoka, M.; Soloshonok, V.A.; Coelho, J.A.S.; Toste, F.D. Modern Approaches for Asymmetric Construction of Carbon-Fluorine Quaternary Stereogenic Centers: Synthetic Challenges and Pharmaceutical Needs. Chem. Rev. 2018, 118, 3887-3964. [CrossRef]

31. Wolstenhulme, J.R.; Gouverneur, V. Asymmetric Fluorocyclizations of Alkenes. Acc. Chem. Res. 2014, 47, 3560-3570. [CrossRef]

32. Ishimaru, T.; Shibata, N.; Horikawa, T.; Yasuda, N.; Nakamura, S.; Toru, T.; Shiro, M. Cinchona Alkaloid Catalyzed Enantioselective Fluorination of Allyl Silanes, Silyl Enol Ethers, and Oxindoles. Angew. Chem. Int. Ed. 2008, 47, 4157-4161. [CrossRef]

33. Rauniyar, V.; Lackner, A.D.; Hamilton, G.L.; Toste, F.D. Asymmetric Electrophilic Fluorination Using an Anionic Chiral Phase-Transfer Catalyst. Science 2011, 334, 1681-1684. [CrossRef]

34. Kong, W.; Feige, P.; de Haro, T.; Nevado, C. Regio- and Enantioselective Aminofluorination of Alkenes. Angew. Chem. Int. Ed. 2013, 52, 2469-2473. [CrossRef] 
35. Wolstenhulme, J.R.; Rosenqvist, J.; Lozano, O.; Ilupeju, J.; Wurz, N.; Engle, K.M.; Pidgeon, G.W.; Moore, P.R.; Sandford, G.; Gouverneur, V. Asymmetric Electrophilic Fluorocyclization with Carbon Nucleophiles. Angew. Chem. Int. Ed. 2013, 52, 9796-9800. [CrossRef] [PubMed]

36. Mennie, K.M.; Banik, S.M.; Reichert, E.C.; Jacobsen, E.N. Catalytic Diastero- and Enantioselective Fluoroamination of Alkenes. J. Am. Chem. Soc. 2018, 140, 4797-4802. [CrossRef] [PubMed]

37. Scheidt, F.; Schäfer, M.; Sarie, J.C.; Daniliuc, C.G.; Molloy, J.J.; Gilmour, R. Enantioselective, Catalytic Vicinal Difluorination of Alkenes. Angew. Chem. Int. Ed. 2018, 57, 16431-16435. [CrossRef]

38. Zhao, J.; Jiang, M.; Liu, J.-T. Synthesis of Fluoromethyl-Substituted Isoxazolines via Transition Metal-Free Oxyfluorination of Alkenyl Oximes. Adv. Synth. Catal. 2017, 359, 1626-1630. [CrossRef]

39. Egami, H.; Asada, J.; Sato, K.; Hashizume, D.; Kawato, Y.; Hamashima, Y. Asymmetric Fluorolactonization with a Bifunctional Hydroxyl Carboxylate Catalyst. J. Am. Chem. Soc. 2015, 137, 10132-10135. [CrossRef] [PubMed]

40. Egami, H.; Niwa, T.; Sato, H.; Hotta, R.; Rouno, D.; Kawato, Y.; Hamashima, Y. Dianionic Phase-Transfer Catalyst for Asymmetric Fluorocyclization. J. Am. Chem. Soc. 2018, 140, 2785-2788. [CrossRef]

41. Niwa, T.; Ujiie, K.; Sato, H.; Egami, H.; Hamashima, Y. Asymmetric Fluorination of Cyclic Tetrasubstituted Alkenes with a Pendant Amide Groups under Dianionic Phase-Transfer Catalysis. Chem. Pharm. Bull. 2018, 66, 920-922. [CrossRef]

42. Begum, S.A.; Terao, J.; Kambe, N. Conversion of $\left(\mathrm{sp}^{3}\right) \mathrm{C}-\mathrm{F}$ Bonds of Alkyl Fluorides to ( $\left.\mathrm{sp}^{3}\right)$ C-Heteroaton (Heteroatom = I, SR, SeR, TeR) Bonds by the Use of Magnesium Reagents Having Heteroatom Substituents. Chem. Lett. 2007, 36, 196-197. [CrossRef]

43. Sorochinsky, A.E.; Aceña, J.L.; Soloshonok, V.A. Self-Disproportionation of Enantiomers of Chiral. Non-Racemic Fluoroorganic Compounds: Role of Fluorine as Enabling Element. Synthesis 2013, 45, 141-152. [CrossRef]

Sample Availability: Samples of the compounds 3 are available from the authors.

(C) 2019 by the authors. Licensee MDPI, Basel, Switzerland. This article is an open access article distributed under the terms and conditions of the Creative Commons Attribution (CC BY) license (http://creativecommons.org/licenses/by/4.0/). 